\title{
Measurement of a band-edge tail in the density of states of a photonic-crystal waveguide
}

\author{
S. R. Huisman, ${ }^{1,{ }^{*}}$ G. Ctistis, ${ }^{1}$ S. Stobbe,${ }^{2}$ A. P. Mosk, ${ }^{1}$ J. L. Herek, ${ }^{1}$ A. Lagendijk,${ }^{1,3}$ P. Lodahl, ${ }^{2}$ \\ W. L. Vos, ${ }^{1}$ and P. W. H. Pinkse ${ }^{1}$ \\ ${ }^{1}$ MESA + Institute for Nanotechnology, University of Twente, P.O. Box 217, AE-7500 Enschede, The Netherlands \\ ${ }^{2}$ Niels Bohr Institute, University of Copenhagen, Blegdamsvej 17, DK-2100 Copenhagen, Denmark \\ ${ }^{3}$ FOM Institute for Atomic and Molecular Physics, Science Park 104, XG-1098 Amsterdam, The Netherlands
}

(Received 3 January 2012; revised manuscript received 21 September 2012; published 26 October 2012)

\begin{abstract}
We investigate light transport near the band edge of slow-light photonic-crystal waveguides using phasesensitive near-field microscopy. We obtain and interpret high-resolution band structures, allowing the retrieval of the optical density of states for a one-dimensional periodic system with weak intrinsic disorder. Because of this disorder, the band edge is smeared out and the van Hove singularity is removed. The density of states shows a decaying "tail" in the band gap corresponding to Anderson-localized modes, as predicted by Lifshitz for solid-state systems.
\end{abstract}

DOI: 10.1103/PhysRevB.86.155154

PACS number(s): 42.25.Dd, 42.70.Qs, 68.37.Uv, 71.55.Jv

Band gaps and accompanying band-edge effects are among the most intriguing phenomena in solid-state physics. ${ }^{1}$ Unavoidable disorder in periodic media strongly alters the transport of electrons, phonons, or photons, ultimately resulting in the breakdown of transport, known as Anderson localization. $^{2-6}$ Electromagnetic waves form an excellent platform to study the effects of disorder on bosonic propagation because of the availability of strong scatterers, high-energy resolution, polarization and phase control, and subwavelength fabrication precision. For many years, microwave experiments have improved our understanding of disorder, such as the demonstration of localized modes near the band edge. ${ }^{7,8}$ However, with current nanofabrication methods, nanophotonic structures have become excellent alternatives for performing experiments at optical energies. This allows us to study the influence of disorder with a direct impact on applied nanophotonics.

Photonic-crystal waveguides are commonly used for strong light confinement with unique dispersion essential for slow light propagation and enhanced light-matter interactions. ${ }^{9-12}$ Light propagation in these waveguides can be approximated by one-dimensional transport. Intrinsic disorder should result in Anderson localized modes ${ }^{13}$ smearing out the band edge and removing the Van Hove singularity. The localized modes in the band gap contribute to the formation of the optical equivalent of a Lifshitz tail in the density of states (DOS). ${ }^{14}$ For doped semiconductors and superconductors, the Lifshitz tail is known as a "tail" in the DOS that decays away from the band edge caused by the ensemble of localized states. ${ }^{15-19}$ Indeed, Anderson localized modes in photonic-crystal waveguides have been demonstrated near the band edge. ${ }^{20-25}$ Furthermore, recent optical experiments demonstrate that the effects of strong scattering can be observed in the DOS..$^{23,26-29}$ However, no experiments near the band edge have been conducted to reveal the optical Lifshitz tail in the DOS. Band-edge phenomena in the DOS have been studied with scanning tunneling microscopy (STM) and spectroscopy (STS) for many years. Surprisingly, to our knowledge, clear identification of a Lifshitz tail is missing, possibly caused by complicated band structures, fabrication control, temperature broadening, and the available energy resolution.
In this article, we combine the optical analog of STM, nearfield scanning optical microscopy (NSOM) ${ }^{30}$ with photoniccrystal waveguides. NSOM offers unique opportunities to measure the wave function and the band structure. ${ }^{25,31-36} \mathrm{We}$ observe, near the band edge for transverse electric (TE)-like waveguide modes, Anderson-localized modes that weakly couple to ballistic transverse magnetic (TM)-like modes that extend over the entire waveguide. The localized modes are seen to explicitly smear out the band edge in the band structure, an observation most relevant for disordered quantum systems. ${ }^{37}$ From the band structure, the DOS is reconstructed, demonstrating the absence of the Van Hove singularity and yielding a direct observation of an optical Lifshitz tail in a one-dimensional (1D) system.

Figure 1(a) illustrates our experiment. A continuous-wave laser (Toptica DL Pro 940) with a tunable wavelength $\lambda$ between 907 and $990 \mathrm{~nm}$ and a linewidth of $0.1 \mathrm{MHz}$ is side-coupled on a cleaved end facet of a GaAs photonic-crystal waveguide (right SEM image) with an objective (NA $=0.55$ ). The incident light is polarized with an angle of approximately $45^{\circ}$ with respect to the normal of the waveguide to excite both TE-like (polarization is oriented in the crystal plane, $E \| y$ ) and TM-like modes (polarization is oriented perpendicular to the crystal plane, $E \| z$ ). The field pattern is collected approximately $200 \mu \mathrm{m}$ away from the coupling facet using an aluminum-coated near-field tip with an aperture of $160 \pm$ $10 \mathrm{~nm}$ (left SEM image). We perform phase-sensitive NSOM using heterodyne detection. ${ }^{38}$

The photonic-crystal waveguide consists of a 1-mm-long photonic-crystal slab with holes forming a triangular lattice with pitch $a=240 \mathrm{~nm}$, a normalized hole radius of $\frac{r}{a}=0.309$, and a slab thickness $h=160 \mathrm{~nm}$. A row of missing holes forms the W1 waveguide. Details on sample fabrication can be found in Refs. 23 and 24, where Anderson localization was demonstrated for waveguides fabricated under identical conditions. Figure 1(b) shows the calculated band structure along the propagation axis for such a photonic-crystal waveguide. ${ }^{39}$ The blue and red bands describe modes that are guided by the line defect for TM- and TE-polarized light, respectively. The blue and pink areas mark continua of modes propagating in the surrounding photonic crystal for TM- and TE-polarized 

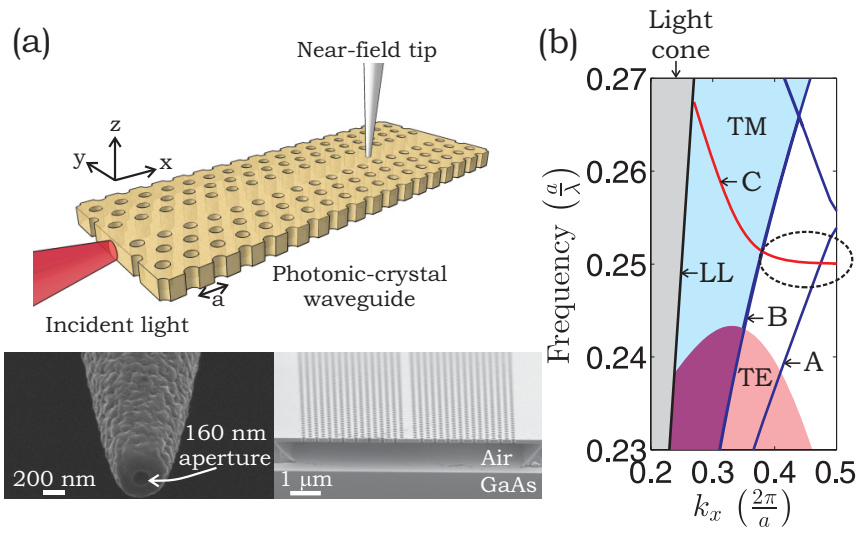

FIG. 1. (Color online) (a) Laser light is side-coupled on a GaAs photonic-crystal waveguide. Light propagation is studied with a near-field tip. SEM images of the coated near-field tip (left) and the waveguide (right) are shown. (b) Calculated band structure showing both TE-like (red) and TM-like (blue) guided modes in the 2D band gap for TE-like modes for $\frac{r}{a}=0.303$ and $\frac{h}{a}=0.67$. The encircled area is the main focus of this article. Here, the TE-like waveguide mode $\mathrm{C}$ becomes flat at the band edge, leading to a Van Hove divergence in the DOS. The black diagonal line represents the light line (LL).

light, respectively, which overlap at the purple area. We concentrate on modes $\mathrm{A}$ and $\mathrm{B}$, which are TM-like, and mode $\mathrm{C}$, which is TE-like. Intrinsic disorder causes Anderson localization in the slow-light regime of mode $\mathrm{C}$ (near $k_{x}=0.5$; encircled area), ${ }^{20,22,24}$ where the dispersion relation flattens and the optical DOS ideally diverges.

Figure 2 presents measured near-field amplitudes at different excitation frequencies. Periodic beating patterns are observed above [Fig. 2(a)] and below [Fig. 2(d)] the band edge of the TE mode (C). Spatial Fourier transforms ${ }^{31}$ and Bloch-mode reconstruction ${ }^{40}$ confirm that these patterns are completely described by a superposition of propagating Bloch modes. ${ }^{35}$ Since these propagating modes are unperturbed by intrinsic disorder and extend over the entire waveguide, they are considered not to be localized and are therefore referred to as propagating. For $907 \leqslant \lambda \leqslant 942.4$ and $954.9 \leqslant \lambda \leqslant$ $990 \mathrm{~nm}$, patterns of propagating Bloch modes similar to those in Figs. 2(a) and 2(d), respectively, were observed.

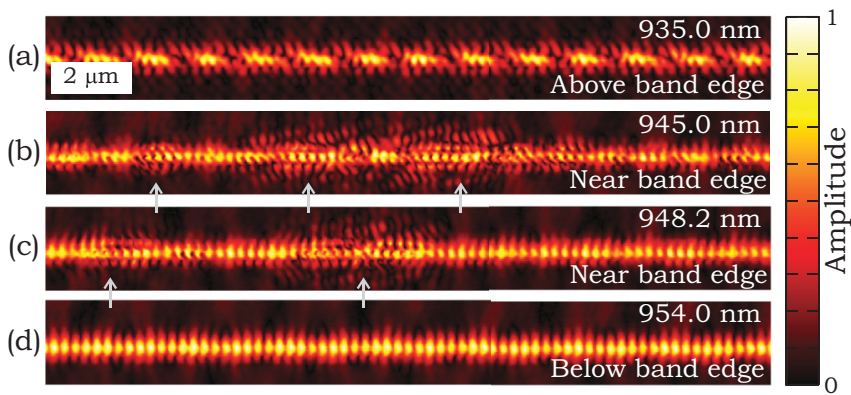

FIG. 2. (Color online) Amplitude for a photonic-crystal waveguide measured at different frequencies above, near, and below the band edge. (a, d) Propagating Bloch modes are shown; (b, c) Anderson-localized random modes are observed. The arrows mark localized states.
Figures 2(b) and 2(c) are measured near the band edge, corresponding to frequencies in the range where the periodic patterns are perturbed by standing-wave field patterns (marked by arrows) that can extend up to approximately $3 a$ into the surrounding crystal. These perturbations are the localized modes expected at the band edge. We have verified that these localized modes occur at random locations along the waveguide only within a wavelength range of $942.4-954.9 \mathrm{~nm}$ near the band edge of mode $\mathrm{C}$. We have confirmed for three waveguides with different $\frac{r}{a}$ values that the frequency range where such localized modes occur follows the shift of the predicted band edge, consistent with previous observations. ${ }^{20}$ The extended field patterns in the surrounding photonic crystal indicate high field amplitudes in the center of the waveguide. The observed maximum amplitude is likely quenched by the presence of the near-field tip. From spatial Fourier transforms we know that the periodic background is formed by TM-like modes (A and B), which contain most of the field energy. ${ }^{35}$ The observed localized modes agree well with calculated profiles of localized modes. ${ }^{14,41}$ Moreover, from Bloch-mode reconstruction ${ }^{40}$ we know that these are not a superposition of Bloch modes. Clearly, from Figs. 2(b) and 2(c) it can be deduced that the presence of the localized modes is strongly wavelength dependent. We have verified that the localized modes indeed have a narrow linewidth [see Figs. 3(a)-3(c) and Ref. 42].

We observe Anderson-localized modes far along the waveguide, where the intensity should naively be vanishingly small. Therefore we explain how the modes are excited. The nearfield patterns in Figs. 3(a)-3(c) were obtained with an incident polarization angle of approximately $45^{\circ}$ with respect to the normal of the photonic-crystal waveguide to excite both TEand TM-like modes. Figure 3(d) shows the near-field pattern at $\lambda=948.6 \mathrm{~nm}$ when the incident polarization angle is $0^{\circ}$ to excite only TM-like modes. We observe an field pattern identical to that in Fig. 3(b). From spatial Fourier transforms, only TM-like Bloch modes were identified, but the localized

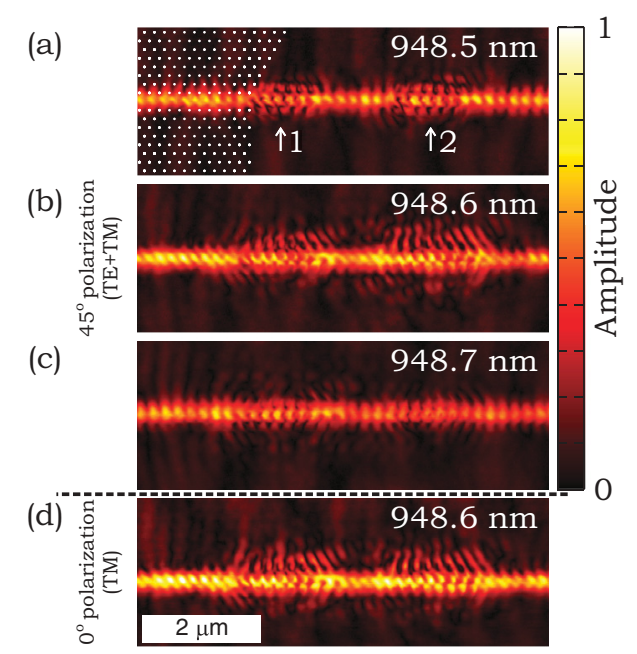

FIG. 3. (Color online) Amplitude for two coupled localized modes (arrows 1 and 2) for three wavelengths separated by $0.1 \mathrm{~nm}$ for two incident polarizations with respect to the pores $\left[(\mathrm{a}-\mathrm{c}) \approx 45^{\circ}\right.$; (d) $0^{\circ} \mathrm{J}$. Amplitudes are normalized to the amplitude of light propagating along the surface of the structure. White circles in (a) indicate the locations of the holes. 
modes remained observed. We conclude that we are detecting a subset of localized modes at the band edge for the TE-like mode $(\mathrm{C})$ that becomes excited by the TM-like propagating mode (A), consistent with previous interpretations. ${ }^{21} \mathrm{We}$ anticipate that the weak coupling between localized modes and ballistic light offers an opportunity to address, manipulate, and read out light-matter interactions with localized modes.

We have collected near-field patterns over the wide spatial range of $x=73 \mu \mathrm{m}$ and $y=2.5 \mu \mathrm{m}$ for a whole range of reduced laser frequencies $\omega=\frac{a}{\lambda}$ and obtained their spatial Fourier transform $S_{\text {int }}\left(k_{x}, y, \omega\right)$. After normalization ${ }^{45}$ this resulted in the experimentally reconstructed band structure shown in Fig. 4(a). ${ }^{31}$ At $0<k_{x}<0.5$ the calculated folded band structure in Fig. 1(b) is overlapped as symbols, showing good agreement with the maxima in $S_{\text {int }}\left(k_{x}, \omega\right)$. One expects that Bloch harmonics repeat every Brillouin zone $\left(k_{x}=k_{0}+\right.$ $n \cdot a, n \in \mathbb{Z})$ and are symmetric around the Bragg conditions $\left(k_{x}=n+0.5, n \in \mathbb{Z}\right)$. The identification of the modes as TE-like (circles) and TM-like (triangles) modes was confirmed
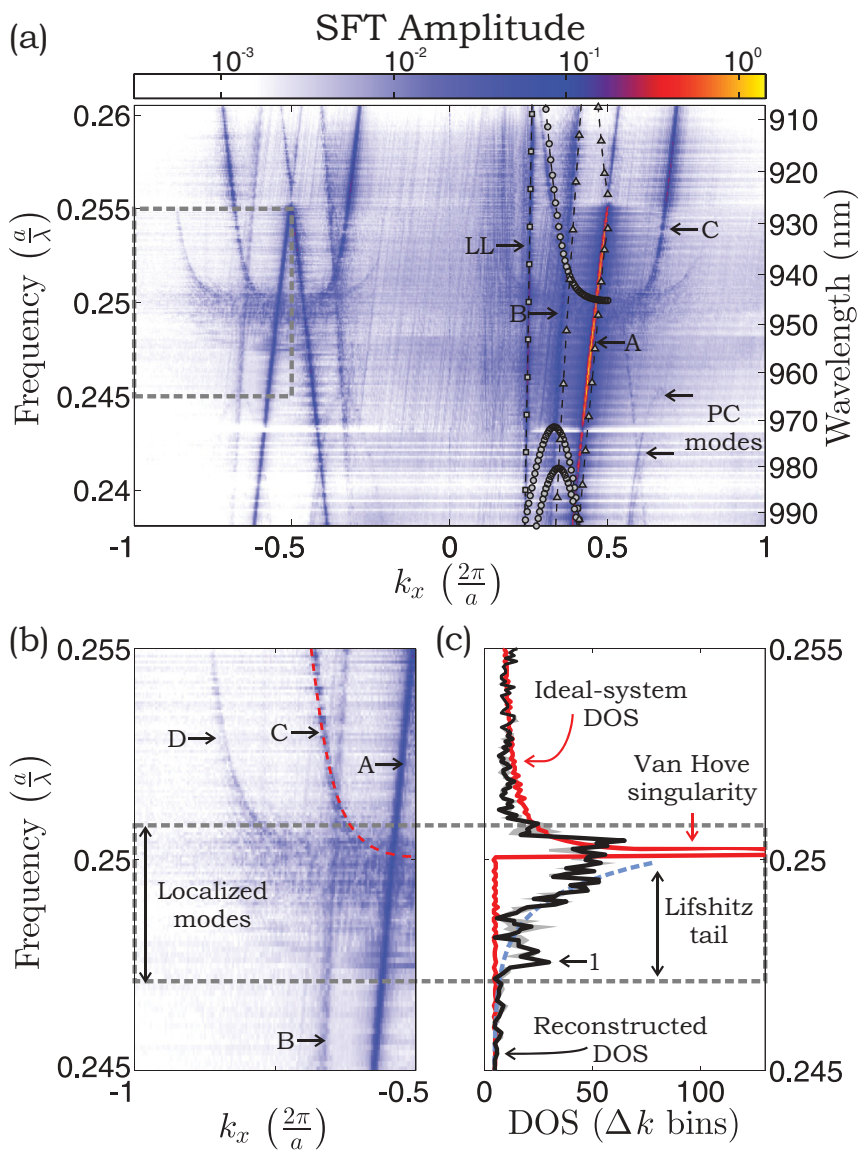

FIG. 4. (Color) (a) Experimentally reconstructed band structure obtained from near-field patterns. The color represents the amplitude of the spatial Fourier (SFT) coefficients $\left|S_{\text {int }}\left(k_{x}, \omega\right)\right|$. Black lines are fitted modes of Fig. 1(b). The gray rectangle is highlighted in (b), where localized modes are represented by the smeared-out band edge. The dashed (red) line approximates mode $\mathrm{C}$ for the ideal system. (c) Experimentally reconstructed DOS (black curve) compared with a fitted DOS (red curve) for an ideal nondisordered waveguide. The single-exponential fit [dashed (blue) line] is a guide for the eye for the Lifshitz tail. by rotating the polarization of the incident light. We identify a narrow stopgap for mode $\mathrm{A}$ at $\omega_{s}=0.2555(k=-0.5,0.5)$, where the character of the modes changes. As a consequence, the overlap with the excitation beam changes and hence the spatial Fourier transform amplitudes of the modes change abruptly around $\omega \approx \omega_{s}$. The band edge for the TE-polarized mode $(\mathrm{C})$ is located at $\omega=0.250(k=-0.5,0.5)$.

In Fig. 4(b) a zoom-in of the band structure is shown near the band edge of mode $\mathrm{C}$ in the range $-1<k_{x}<-0.5$. A most intriguing feature is that our measurements do not show a sharp band edge, but a softened cutoff, in agreement with predictions of Ref. 14. The smearing-out of the cutoff in the range $0.247<$ $\omega<0.251$ is caused by localized states and starts to appear at a group index of $n_{g}(\omega=0.251)=37$. No localized modes are observed outside this cutoff region. The width of the blurred cutoff $\Delta \omega=0.004$ is a measure for the amount of disorder. If variations in the hole size and position are the dominant source of disorder, ${ }^{14}$ this would indicate a standard deviation of $\sigma=$ $0.015 a$ in the hole positioning and hole radius, in reasonable agreement with sample characterization. The group index at which we first observe localized modes is consistent with our previous estimations. ${ }^{23}$ Mode $\mathrm{D}$ is not a Bloch mode, but likely the result of a third-order scattering process, since its wave vector is given by $2 k_{C}-k_{A} \cdot{ }^{46}$ Also, higher order modes like $3 k_{A}$ are observed (not shown here). We suspect that these additional modes are caused by the $\chi^{3}$ nonlinearity of GaAs. This therefore is a potential system for study of the relation between nonlinear transport and disorder.

Figure 4(c) shows the main result of this article: We have reconstructed the DOS from the bands in Fig. 4(b). For each $\omega$ we have calculated the number of $k_{x}$ bins that satisfy $\left|S_{\text {int }}\left(k_{x}, \omega\right)\right|>q$, with $\Delta k_{x}=0.0033$ and threshold $q=0.08$. This measure for the DOS is shown in Fig. 4(c) (black curve). The extra mode (D) is not taken into account. The shape of the reconstructed DOS is not very sensitive to the exact value of $q$, as indicated by the gray area behind the curve, indicating the reconstructed DOS in the range $0.06<q<0.1$. We have applied a similar sampling method to the calculated band structure in Fig. 1(b) to modes A, B, and C, representing the calculated DOS of an ideal periodic waveguide (red curve). This DOS is scaled to have the same value as the experimental DOS in the range $0.255>\omega>0.254$. Both the experimental and the calculated DOS are approximately constant in the range $0.255>\omega>0.251$ and for $0.247>\omega$. For these frequency ranges no localized modes are observed. Note the contribution of the TM-like modes, which lead to a finite DOS for $0.247>\omega$. In the range $0.251>\omega>0.250$ both the experimental and the calculated DOS increase rapidly. The DOS of an ideal periodic system diverges to infinity as $\rho(\omega) \propto\left(\omega-\omega_{\text {gap }}\right)^{-\frac{1}{2}}$, forming the Van Hove singularity. ${ }^{47,48}$ The experimental DOS follows this increase, until it saturates at $\omega=0.2505$. In this range the first localized modes are observed. Although the single peak at $\omega=0.2476$ (arrow 1) belongs to a single localized mode, it is unclear whether the sharp features in the reconstructed DOS are dominated by individual localized states, since localized states are present at every $\omega$ setting in this range. In future this could be answered by reconstructing band structures with a smaller $\Delta \omega$. It is clear, though, that the Van Hove divergence is absent, in agreement with computations by Savona. ${ }^{14}$ In the band gap for 
$0.250>\omega>0.247$ the experimental and the calculated DOS differ significantly: The calculated DOS is constant, whereas the experimental DOS slowly decays away from the band edge, forming the Lifshitz tail known from solid-state systems. ${ }^{15-19}$ Our present data do not allow us to draw conclusions about the exact shape of the tail, which is debated in the literature, but show the possibility of addressing this issue in future experiments.

We have reported near-field measurements of localized modes using phase-sensitive NSOM; the experimentally obtained band structure reveals how the localized states perturb the band edge. Ensemble averaging by measuring band structures of different parts of the sample should smooth the envelope of the reconstructed DOS and will afford the possibility of quantitatively studying the shape of the Lifshitz tail. We also predict that the Lifshitz tail should appear when the DOS is directly probed by studying the emission of embedded quantum dots. ${ }^{11,49}$ Ensemble averaging for several degrees of disorder will allow us to study the scaling properties of localized states near the band edge..$^{50}$

We thank J. Bertolotti, D. Dikken, H. J. W. Zandvliet, P. D. García, L. Kuipers, and H. Thyrrestrup for stimulating discussions and C. Harteveld, J. P. Korterik, and F. Segerink for technical support. This work was supported by FOM and NWO-Nano.
*Correspondence author: s.r.huisman@utwente.nl; www.utwente.nl/ mesaplus/anp

${ }^{1}$ N. W. Ashcroft, and N. D. Mermin, Solid State Physics (Thomson Learning, New York, 1976).

${ }^{2}$ P. W. Anderson, Phys. Rev. 109, 1492 (1958).

${ }^{3}$ B. L. Altshuler, V. E. Kravtsov, I. V. Lerner, in Mesoscopic Phenomena in Solids, edited by B. L. Altshuler, P. A. Lee, and R. A. Webb (North-Holland, Amsterdam, 1991).

${ }^{4} \mathrm{P}$. Sheng, Introduction to Wave Scattering, Localization, and Mesoscopic Phenomena (Academic Press, San Diego, CA, 1995).

${ }^{5}$ E. Akkermans and G. Montambaux, Mesoscopic Physics of Electrons and Photons (Cambridge University Press, Cambridge, 2007).

${ }^{6}$ A. Lagendijk, B. van Tiggelen, and D. S. Wiersma, Phys. Today 62, 24 (2009).

${ }^{7}$ M. Stoytchev and A. Z. Genack, Phys. Rev. B 55, R8617 (1997).

${ }^{8}$ A. A. Chabanov, M. Stoytchev, and A. Z. Genack, Nature (London) 404, 850 (2000).

${ }^{9}$ T. F. Krauss, J. Phys. D: Appl. Phys. 40, 2666 (2007).

${ }^{10}$ J. D. Joannopoulos, S. G. Johnson, J. N. Winn, and R. D. Meade, Photonic Crystals, Molding the Flow of Light (Princeton University Press, Princeton, NJ, 2008).

${ }^{11}$ T. Lund-Hansen, S. Stobbe, B. Julsgaard, H. Thyrrestrup, T. Sünner, M. Kamp, A. Forchel, and P. Lodah, Phys. Rev. Lett. 101, 113903 (2008).

${ }^{12}$ L. O’Faolain, D. M. Beggs, T. P. White, T. Kampfrath, L. Kuipers, and T. F. Krauss, IEEE Photon. J. 2, 404 (2010).

${ }^{13}$ S. John, Phys. Rev. Lett. 58, 2486 (1987).

${ }^{14}$ V. Savona, Phys. Rev. B 83, 085301 (2011).

${ }^{15}$ I. M. Lifshitz, Adv. Phys. 13, 483 (1964).

${ }^{16}$ R. J. Elliott, J. A. Krumhansl, and P. L. Leath, Rev. Mod. Phys. 46, 465 (1974).

${ }^{17}$ P. Van Mieghem, Rev. Mod. Phys. 64, 755 (1992).

${ }^{18}$ A. V. Balatsky, I. Vekhter, and J.-X. Zhu, Rev. Mod. Phys. 78, 373 (2006).

${ }^{19}$ M. Aizenman and S. Warzel, Phys. Rev. Lett. 106, 136804 (2011).

${ }^{20}$ J. Topolancik, B. Ilic, and F. Vollmer, Phys. Rev. Lett. 99, 253901 (2007).

${ }^{21}$ J. Topolancik, F. Vollmer, R. Ilic, and M. Crescimanno, Opt. Express 17, 12470 (2009).

${ }^{22}$ N. Le Thomas, H. Zhang, J. Jágerská, V. Zabelin, R. Houdré, I. Sagnes, and A. Talneau, Phys. Rev. B 80, 125332 (2009).

${ }^{23}$ L. Sapienza, H. Thyrrestrup, S. Stobbe, P. D. García, S. Smolka, and P. Lodahl, Science 327, 1352 (2010).
${ }^{24}$ P. D. García, S. Smolka, S. Stobbe, and P. Lodahl, Phys. Rev. B 82, 165103 (2010).

${ }^{25}$ M. Spasenović, D. M. Beggs, P. Lalanne, T. F. Krauss, and L. Kuipers, preceding paper, Phys. Rev. B 86, 155153 (2012).

${ }^{26}$ P. V. Ruijgrok, R. Wüest, A. A. Rebane, A. Renn, and V. Sandoghdar, Opt. Express 18, 6360 (2010).

${ }^{27}$ M. D. Birowosuto, S. E. Skipetrov, W. L. Vos, and A. P. Mosk, Phys. Rev. Lett. 105, 013904 (2010).

${ }^{28}$ V. Krachmalnicoff, E. Castanié, Y. De Wilde, and R. Carminati, Phys. Rev. Lett. 105, 183901 (2010).

${ }^{29}$ R. Sapienza, P. Bondareff, R. Pierrat, B. Habert, R. Carminati, and N. F. van Hulst, Phys. Rev. Lett. 106, 163902 (2011).

${ }^{30}$ L. Novotny and B. Hecht, Principles of Nano-Optics (Cambridge University Press, Cambridge, 2006).

${ }^{31}$ H. Gersen, T. J. Karle, R. J. P. Engelen, W. Bogaerts, J. P. Korterik, N. F. van Hulst, T. F. Krauss, and L. Kuipers, Phys. Rev. Lett. 94, 073903 (2005)

${ }^{32}$ N. Louvion, A. Rahmani, C. Seassal, S. Callard, D. Gérard, and F. de Fornel, Opt. Lett. 31, 2160 (2006).

${ }^{33}$ S. Mujumdar, A. F. Koenderink, T. Sünner, B. C. Buchler, M. Kamp, A. Forchel, and V. Sandoghdar, Opt. Express 15, 17214 (2007).

${ }^{34}$ S. Ha, M. Spasenović, A. A. Sukhorukov, T. P. White, C. M. de Sterke, L. Kuipers, T. F. Krauss, and Y. S. Kivshar, J. Opt. Soc. Am. B 28, 955 (2011).

${ }^{35}$ S. R. Huisman, G. Ctistis, S. Stobbe, J. L. Herek, P. Lodahl, W. L. Vos, and P. W. H. Pinkse, J. Appl. Phys. 111, 033108 (2012).

${ }^{36}$ F. Riboli, P. Barthelemy, S. Vignolini, F. Intonti, A. De Rossi, S. Combrie, and D. S. Wiersma, Opt. Lett. 36, 127 (2011).

${ }^{37}$ B. Altshuler, B. Doucot, M. Mézard, and G. Shlyapnikov, Organizers, Conference on Disordered Quantum Systems, Paris, June 18-22, 2012.

${ }^{38}$ M. L. M. Balistreri, J. P. Korterik, L. Kuipers, and N. F. van Hulst, Phys. Rev. Lett. 85, 294 (2000).

${ }^{39}$ S. G. Johnson and J. D. Joannopoulos, Opt. Express 8, 173 (2001).

${ }^{40}$ S. Ha, A. A. Sukhorukov, K. B. Dossou, L. C. Botten, C. M. de Sterke, and Y. S. Kivshar, Opt. Lett. 34, 3776 (2009).

${ }^{41}$ S. Smolka, H. Thyrrestrup, L. Sapienza, T. B. Lehmann, K. R. Rix, L. S. Froufe-Pérez, P. D. García, and P. Lodahl, New J. Phys. 13, 063044 (2011)

${ }^{42}$ Localized modes in photonic-crystal waveguides are known to be narrow-band. ${ }^{14,20-24,41}$ In Fig. 3 we show the wavelength dependence of two such modes (1 and 2) with a resolution of $\Delta \lambda=0.1 \mathrm{~nm}$. 
In Fig. 3(a) we observe that the periodic pattern is perturbed at the locations of the arrows at $\lambda=948.5 \mathrm{~nm}$. In Fig. 3(b) we observe the localized mode at $\lambda=948.6 \mathrm{~nm}$, where the perturbations extend maximally in the surrounding photonic crystal. In Fig. 3(c), at $\lambda=948.7 \mathrm{~nm}$, the localized modes are suppressed. At $\lambda=948.9 \mathrm{~nm}$ the localized modes have completely vanished (not shown). This demonstrates that these localized modes have a linewidth of $\Delta \lambda \approx 0.2 \mathrm{~nm}$, corresponding to $Q \sim 10^{3}-10^{4}$. We have observed $\sim 10^{2}$ localized modes and they fully extend within a range of typically $\Delta \lambda<0.5 \mathrm{~nm}$. The observed linewidths should be considered the upper limit for the true resonance widths, since near-field tips are known to shift and broaden resonances. ${ }^{33,43,44}$

${ }^{43}$ W. C. L. Hopman, K. O. van der Werf, A. J. F. Hollink, W. Bogaerts, V. Subramaniam, and R. M. de Ridder, Opt. Express 14, 8745 (2006).
${ }^{44}$ L. Lalouat, B. Cluzel, P. Velha, E. Picard, D. Peyrade, J. P. Hugonin, P. Lalanne, E. Hadji, and F. de Fornel, Phys. Rev. B 76, 041102 (2007).

${ }^{45}$ We have calculated the SFT $S(k, y, \omega)$ for each line parallel to the line defect. We define $S_{\text {int }}(k, \omega)=\int|S(k, y, \omega)| d y$. All $S_{\text {int }}(k, \omega)$ values are normalized to have the same average value for $-3.5<$ $k_{x}<-1.8$, which is dominated by $\frac{1}{f}$ noise.

${ }^{46}$ One has to take mode $k_{A}$ in the range $-0.5<k_{x}<0$ and mode $k_{C}$ in the range $-1.0<k_{x}<-0.5$.

${ }^{47}$ S. John and T. Quang, Phys. Rev. A 50, 1764 (1994).

${ }^{48}$ Z. Y. Li and Y. Xia, Phys. Rev. A 63, 043817 (2001).

${ }^{49}$ Q. Wang, S. Stobbe, and P. Lodahl, Phys. Rev. Lett. 107, 167404 (2011); M. D. Leistikow, A. P. Mosk, E. Yeganegi, S. R. Huisman, A. Lagendijk, and W. L. Vos, ibid. 107, 193903 (2011).

${ }^{50}$ L. I. Deych, D. Zaslavsky, and A. A. Lisyansky, Phys. Rev. Lett. 81, 5390 (1998). 